\title{
Embedding-Based Sliding Mode Control for Linear Time Varying Systems
}

\author{
Mohammad Reza Zarrabi ${ }^{1}$, Mohammad Hadi Farahi ${ }^{1}$, Ali Jafar Koshkouei ${ }^{2}$, Sohrab Effati ${ }^{1}$, \\ Keith Burnham ${ }^{2}$ \\ ${ }^{1}$ Department of Applied Mathematics, Ferdowsi University of Mashhad, Mashhad, Iran \\ ${ }^{2}$ Control Theory and Applications Centre, Coventry University, Coventry, UK \\ E-mail:s-effati@um.ac.ir
}

Received February 20, 2011; revised March 10, 2011; accepted March 13, 2011

\begin{abstract}
In this paper, a novel strategy using embedding process and sliding surface is proposed. In this method, a state trajectory starting from a given initial point reaches a definite point on a sliding surface in the minimum time, and then tends to the origin along the sliding surface (SS). In the first, a SS is designed, then using an appropriate measure, an embedding is constructed to solve a time optimal control problem such that the system trajectory reaches the SS in minimum time, after that a control is designed such that the system trajectory tends to the origin along the SS. It is well-known that the main disadvantage of the use of sliding mode controls (SMCs) is a phenomenon, the so-called chattering. The proposed SMC here is piecewise continuous and chattering free. Some numerical examples is presented to illustrate the effectiveness and reliability of the proposed method.
\end{abstract}

Keywords: Time Optimal Control Problem, Measure Theory, Sliding Mode Control, Sliding Surface Design, Equivalent Control

\section{Introduction}

The concept of sliding mode control (SMC) introduced a second order system by Emel'yanov [1] in the late 1960s based on the conceptions of variable structure control (VSC) in which the second order system trajectories has been driven towards a line in the state space termed as the sliding line and enforcing the trajectories to the origin. However, Flügge-Lotz [2] was the first to present the concept of sliding motion, and Filippov [3] was the first to consider the solution of differential equations with a discontinuous right-hand side. Filippov's pioneering work still serves as the basis for work in sliding mode control which was essentially developed by Utkin [4] and Emel'yanov [1,5], Draženović [6] and their co-workers.

The pioneering work had not been presented the outside of Russia until the mid 1970s when a book by Itkis [7] and a survey paper by Utkin [8] were published in the west. SMC which is a particular type of control, known as variable structure control (VSC), is a powerful and robust control, and it has been extensively studied in the last three decades for many classes of linear and nonli- near systems, from theoretical concepts to industrial applications, including autonomous underwater robot [9], continuously stirred tank reactor [10], PUMA 560 robot [11], finger for a prosthetic hand [12], cable suspended loads [13] and four rotors helicopter [14].

Utkin [4] has developed the concept of sliding mode control to guarantees the existence of a sliding mode motion. In the classical SMC approach, infinite frequency control switching between different sub-controllers is required to maintain the trajectories on a prespecified surface in the state space and to eventually enforce the state trajectories to the equilibrium point along the surface. Therefore, the system stability and behaviour depend on the selection of sliding surface $[8,15]$. Several methods have been proposed to design a stable SS, such neural network [9], fuzzy SMC [16], SMC a system with mismatched uncertainties [17], discrete-time SMC [18], minimizing integral absolute error [19], passivity-based SMC $[10,20]$ and flatness, back stepping with SMC [21]. SMC approach is well known as one of powerful and effective robust control approaches to reject the matched disturbances, to reduce the influence of unmatched uncertainties and insensitivity to parameter variations. How- 
ever, the repetitious control switching creates a phenomenon, the so-called chattering, which may be dangerous and exhibits high-frequency vibrations of the controlled plant $[22,23]$. To overcome this unwanted behaviour, many methods including several continuous approximations of the discontinuous SMCs have been proposed, in which the switching function enforces the trajectories to stay within a boundary layer of the sliding surface. The main disadvantage of such approximation is that the robustness of SMC may be lost inside the boundary layer if the size of the boundary layer is not sufficiently small. In fact, the uncertainties and disturbances may influence the system behaviour. Other methods for eliminating the chattering are based on high order sliding mode control concepts in which the higher order time derivative of the switching function is used. The traditional discontinuous sliding mode control enforces the state trajectories on the sliding surface while the continuous part of sliding mode control imposes the trajectories remain on the surface after a finite time. The continuous part has a particular structure which leads to the invariance conditions for the sliding motion and in the average sense; it is termed the equivalent control.

On the other hand, many methods based on sliding mode and output feedback control schemes have been also proposed for robust stabilisation of uncertain systems [24,25]. El-Khazali and DeCarlo [25] have designed sliding surfaces for linear time-invariant systems without disturbances using eigenvalue assignment and eigenvector techniques. Żak and Hui [24] have developed a geometric condition to guarantee the existence of a SS and the stability of the reduced order sliding motion. Most established results are based on the matched disturbances, i.e. the disturbances acts in the channels of the inputs. Even when there are uncertainties in the system with unknown structure, SMC is an appropriate control design method. When the system is in a sliding mode, the dimension of the system is reduced and it is the same the SS. This subsystem is termed the reduced-order system, and the stability of the original system depends on the stability of this subsystem.

In this paper, a novel strategy based on measure theory and sliding surface is proposed to design an almost time optimal control as well as a sliding mode control to enforce the system trajectories from an initial point to a definite point on the designed SS in minimum time, and then force the trajectories tend to the equilibrium points along the SS. In fact, the entire of control design is completed in three steps. In the first step, the SS is designed. In the second step, the minimum time optimal control problem from an initial point to a given point on the SS, is solved. In the third step, an equivalent control is designed such that the system trajectories tend to the equi- librium point along the SS. In fact, the trajectory moves along the SS and eventually reaches the equilibrium point (say origin) in infinite-horizon. The proposed controller is piecewise continuous and yields a chattering free motion. Furthermore, this method guarantees a sliding mode motion and system stability. Moreover, the proposed approach is straightforward without requiring any predication or conditions on the initials and using any iterative algorithm.

This paper is organised as follows: Section 2 briefly introduces SMC. Section 3 addresses the functional space and measure theory facts which are used in this paper. Section 4 addresses the proposed SMC approach. In Section 5, some examples are presented to illustrate the procedure and validity of the proposed control design. Finally, conclusions are given in Section 6.

\section{SMC Design}

Consider the linear time varying system

$$
\dot{x}(t)=\boldsymbol{A}(t) x(t)+\boldsymbol{B}(t) u(t),
$$

$x(0)=x_{0}$, and the equilibrium point is the origin, where $\boldsymbol{A} \in \mathbb{R}^{n \times n}, \quad \boldsymbol{B} \in \mathbb{R}^{n \times m}$, while $x(t) \in \mathcal{A}$ is the state and $u(t) \in \mathcal{U}$ is the control input. The sets $\mathcal{A}$ and $\mathcal{U}$ are bounded, closed subsets respectively in $\mathbb{R}^{n}$ and $\mathbb{R}^{m}$. It is assumed that $1 \leq m<n$ and the input distribution matrix $B$ has full rank. Define the sliding function as:

$$
s(x)=G x,
$$

where $G \in \mathbb{R}^{m \times n}$ and the sliding surface as:

$$
S=\left\{x \in \mathbb{R}^{n}: s(x)=0\right\} .
$$

It is also assumed that $G B(t)$ is a nonsingular matrix.

Suppose there exists a finite time $t_{s}$ such that the solution of (2.1) represented by $x(t)$ satisfies

$$
s(x(t))=0 \text { for all } t \geq t_{s},
$$

then an ideal sliding motion is said to be taking place for all $t \geq t_{s}$. Time $t_{s}$ is termed the reaching time and it is the time that the system trajectories (2.1) lie on the surface (2.3). This fact can be mathematically expressed as $s(t)=G x(t)=0$ and $\dot{s}(t)=G x(t)=0$ for all $t \geq t_{s}$.

In this paper an almost optimal control $\left(u_{a o}\right)$ is designed to ensure the trajectories hits the SS in minimum time. Then using the sliding mode conditions $s(t)=0$ and $\dot{s}(t)=0$ as two extra boundary conditions, a subcontrol $\left(u_{e q}\right)$ is designed such that the trajectories converge to the equilibrium point. Thus the traditional SMC is defined as:

$$
u=\left\{\begin{array}{llc}
u_{a o} & \text { if } & 0 \leq t \leq t_{s} \\
u_{e q} & \text { if } & t>t_{s}
\end{array}\right.
$$


where $t_{s}$ is the reaching time to the $S S$, i.e. $S\left(t_{s}\right)=0$. In fact, $u_{a o}$ is a sub-controller which impose the trajectory that moves from an initial point hits the sliding surface at point $C$ in minimum time and $u_{e q}$ is the equivalent control which forces the trajectory moves from $C$ to the equilibrium point along SS.

The sliding surface is selected such that the reducedorder system, i.e. the system during the sliding mode, is stable and crosses from the point $C$. To find the behaviour of the sliding mode system, the system may be decomposed into two subsystems as it is presented here.

If the states are partitioned so that

$$
x=\left[\begin{array}{l}
x_{1} \\
x_{2}
\end{array}\right]
$$

where $x_{1} \in \mathbb{R}^{n-m}$ and $x_{2} \in \mathbb{R}^{m}$, then the linear system (2.1) can be written as the following regular form

$$
\begin{gathered}
\dot{x}_{1}=\boldsymbol{A}_{11}(t) x_{1}(t)+\boldsymbol{A}_{12}(t) x_{2}(t) \\
\dot{x}_{2}=\boldsymbol{A}_{21}(t) x_{1}(t)+\boldsymbol{A}_{22}(t) x_{2}(t)+\boldsymbol{B}_{2}(t) u(t),
\end{gathered}
$$

where the sub-matrices $\boldsymbol{A}_{11}(t), \boldsymbol{A}_{12}(t), \boldsymbol{A}_{21}(t)$, $\boldsymbol{A}_{22}(t)$, and $\boldsymbol{B}_{2}(t)$ are defined appropriately. (See [26] for more details)

Equation (2.6), which is independent of the control, considers as a system that shows sliding motion (the reduced-order system or the system in the sliding mode).

In the next section, we briefly introduce the functional space used in embedding method.

\section{Functional Space}

To describe our embedding method, consider the general following control system:

$$
\begin{aligned}
\dot{x}(t) & =g(t, x, u) \\
\text { s.t. } \quad x(a) & =x_{a}, x(b)=x_{b},
\end{aligned}
$$

where $a$ and $b$ are two nonnegative numbers with $a \leq b$, $t \in(a . b) \subseteq \mathbb{R}$, the trajectory function $x($.$) satisfies$ $x(t) \in \mathcal{A}$ and is absolutely continuous, the control function $u($.$) is Lebesgue measurable and u(t) \in \mathcal{U}$, where $\mathcal{A}$ and $\mathcal{U}$ are respectively closed, and bounded subsets in $\mathbb{R}^{n}$ and $\mathbb{R}^{m}$, and $g:[a, b] \times \mathcal{A} \times \mathcal{U} \rightarrow \mathbb{R}^{n}$ is a continuous time varying vector function.

Clearly the operating regions $\mathcal{A}$ and $\mathcal{U}$ are compact sets and $x_{a}, x_{b} \in \mathcal{A}$. It is desired to design the control $u$ such that the system trajectory starting from the initial state $x_{a}$ reaches the final state $x_{b}$ at $t=b$.

An optimal control problem is presented as the following: Minimise:

$$
J(x, u)=\int_{a}^{b} f_{0}(t, x, u) \mathrm{d} t
$$

subject to:

$$
\begin{gathered}
\dot{x}(t)=g(t, x, u) \\
x(a)=x_{a}, x(b)=x_{b},
\end{gathered}
$$

where $f_{0}:[a, b] \times \mathcal{A} \times \mathcal{U} \rightarrow \mathbb{R}$ is a continuous function.

The pair $w=(x(\cdot), u(\cdot))$ satisfying the conditions (3.4) and (3.5) is termed admissible. The set of all admissible pairs, is denoted by $W$. Now, one seeks to find an optimal pair $w^{*}=\left(x^{*}(\cdot), u^{*}(\cdot)\right) \in W$ which gives the minimum value of $J(x, u)$ defined in (3.3). In general the minimisation of the functional (3.3) over $W$ may not always possible. The set $W$ may be empty, even if $W$ is not a null set, the functional measuring the performance of the system may not achieves its minimum in this set. It appears that the situation may become more promising if the set $W$ could somehow be made larger. In the following a transformation is used to enlarge the set $W$. Let $w=(x(\cdot), u(\cdot))$ be an admissible pair and $B$ an open ball containing $I \times \mathcal{A}$ where $I=[a, b]$. Let $\Phi \in C^{\prime}(B)$ where $C^{\prime}(B)$ denotes the space of realvalued continuously differentiable functions on $B$. Define the function $\Phi^{g}$ as

$$
\begin{aligned}
& \Phi^{g}(t, x(t), u(t))=\frac{\mathrm{d}}{\mathrm{d} t} \Phi(t, x(t)) \\
& =\Phi_{x}(t, x(t)) g(t, x(t), u(t))+\Phi_{t}(t, x(t)),
\end{aligned}
$$

with $(t, x(t), u(t)) \in \Omega=I \times \mathcal{A} \times \mathcal{U}$ for all $t \in I$. The function $\Phi^{g}$ is in the space $C(\Omega)$, the set of all continuous functions on the compact set $\Omega$. For each admissible pair,

$$
\begin{aligned}
& \int_{a}^{b} \Phi^{g}(t, x(t), u(t)) \mathrm{d} t=\Phi(b, x(b))-\Phi(a, x(a))=\Delta \Phi \\
& \forall \Phi \in C^{\prime}(B) .
\end{aligned}
$$

Let $D\left(I^{0}\right)$ be the space of infinitely differentiable real-valued functions with a compact support in $I^{0}$, where $I^{0}=(a, b)$. Define

$$
\begin{aligned}
& \psi_{j}(t, x(t), u(t))=\frac{\mathrm{d}}{\mathrm{d} t}\left(\psi(t) x_{j}(t)\right) \\
& =\psi^{\prime}(t) x_{j}(t)+\psi(t) g_{j}(t, x(t), u(t)), \\
& \forall \psi \in D\left(I^{0}\right), j=1,2, \cdots, n .
\end{aligned}
$$

Then, if $w=(x(\cdot), u(\cdot))$ is an admissible pair, for every $\psi \in D\left(I^{0}\right)$,

$$
\int_{a}^{b} \psi_{j}(t, x(t), u(t)) \mathrm{d} t=0, j=1,2, \cdots, n .
$$

Let $C_{1}(\Omega)$ be a subspace of the space $C(\Omega)$ of all bounded continuous functions on $\Omega$ depending only on the variable $t$. Now, by selecting the function $f \in C_{1}(\Omega)$, it is obtained 


$$
\int_{a}^{b} f(t, x(t), u(t)) \mathrm{d} t=a_{f} \quad\left(f \in C_{1}(\Omega)\right),
$$

the set of Equalities (3.7), of which we have singled out the special cases (3.9) and (3.10), are properties of the admissible pairs in the classical formulation of the optimal control problem. In the following a transformation is developed to a non-classical problem to obtain enhanced properties in some aspects (see [27] for the details).

For each admissible pair $w$, there is a positive linear continuous functional $\Lambda_{w}$ on $C(\Omega)$ such that

$$
\Lambda_{w}: F \rightarrow \int_{I} F(t, x(t), u(t)) \mathrm{d} t \quad(F \in C(\Omega)) .
$$

By the Riesz representation theorem (see [28]) there exists a unique positive Borel measure $\mu$ on $\Omega$ such that

$$
\begin{aligned}
& \Lambda_{w}(F) \rightarrow \int_{I} F(t, x(t), u(t)) \mathrm{d} t=\int_{\Omega} F \mathrm{~d} \mu \equiv \mu(F) \\
& (F \in C(\Omega)) .
\end{aligned}
$$

Thus, the optimal control problem (3.3)-(3.5) is equivalent to the minimisation of

$$
J(\mu)=\int_{\Omega} f_{0} \mathrm{~d} \mu \equiv \mu\left(f_{0}\right) \in \mathbb{R}
$$

over the set of measures $\mu$, associated with the admissible pair $w$, which satisfy

$$
\begin{aligned}
& \mu\left(\Phi^{g}\right)=\Delta \Phi, \Phi \in C^{\prime}(B) \\
& \mu\left(\psi_{j}\right)=0, \psi \in D\left(I^{0}\right), j=1,2, \cdots, n \\
& \mu(f)=a_{f}, f \in C_{1}(\Omega) .
\end{aligned}
$$

Let $M^{+}(\Omega)$ be the set of all positive Borel measures on $\Omega$. Define the set $Q \subseteq M^{+}(\Omega)$ of all positive Borel measures on $\Omega$ which satisfy (3.13). Now if the space $M^{+}(\Omega)$ is topologised by the weak ${ }^{*}$-topology, it can be shown that $Q$ is compact with the topology induced (see [28]). In the sense of the weak ${ }^{*}$-topology, the functional $\mu\left(f_{0}\right): Q \rightarrow \mathbb{R}$ defined as in (3.12), is a linear continuous functional on the compact set $Q$. Thus it attains its minimum on $Q$, and therefore, the measuretheoretical problem, which consists of finding the minimum of the functional (3.12), over the subset of $M^{+}(\Omega)$, possesses a minimising solution, say $\mu^{*}$, in $Q$.

The set $\Omega=I \times \mathcal{A} \times \mathcal{U}$ is covered with a grid, where the grid will be defined by taking all points in $\Omega$ as $z_{j}=\left(t_{j}, x_{j}, u_{j}\right)$. Instead of the infinite-dimensional linear programming problem (3.12) and (3.13), the following finite dimensional linear programming (LP) problem is considered where $z_{j} \in \omega$ in which $\omega$ is an approximately dense subset of $\Omega$ (see [29] and its references for more details). The finite dimensional LP problem, which approximates the action of the infinite dimensional LP problem (3.12) and (3.13) for a sufficient large integer $\mathrm{N}$ is as follows.

$$
\min \sum_{j=1}^{N} \beta_{j} f_{0}\left(z_{j}\right)
$$

s.t.

$$
\begin{aligned}
& \sum_{j=1}^{N} \beta_{j} \Phi_{i}^{g}\left(z_{j}\right)=\Delta \Phi_{i}, i=1,2, \cdots, M_{1}, \Phi_{i} \in C^{\prime}(B) \\
& \sum_{j=1}^{N} \beta_{j} \psi_{r}\left(z_{j}\right)=0, r=1,2, \cdots, M_{2}, \psi \in D\left(I^{0}\right) \\
& \sum_{j=1}^{N} \beta_{j} f_{s}\left(z_{j}\right)=a_{f s}, \quad s=1,2, \cdots, L, f_{s} \in C_{1}(\Omega) \\
& \beta_{j} \geq 0, z_{j} \in \omega, j=1,2, \cdots, N
\end{aligned}
$$

Now, using the solution of this problem, one can obtain the coefficients $\beta_{j}(j=1,2, \cdots, N)$, and also from the analysis of the problem as in Rubio (see [27]), it is possible to obtain the piecewise-constant control function $u(\cdot)$ which approximates the action of the optimal measure. As a final stage, from the dynamical system (3.4) and (3.5), one can obtain the state trajectory $x(\cdot)$.

\section{Applying Embedding Method}

In this section a time optimal control, a sliding surface, and a so-called equivalent control, for a system such as (2.1) is considered.

\subsection{Step 1: Sliding Surface Design}

Consider the regular form of the linear dynamical system (2.6) and (2.7). Define the sliding surface (2.3) as:

$$
s(x)=\left(\boldsymbol{I}+\boldsymbol{A}_{11}\right) x_{1}+\boldsymbol{A}_{12} X_{2}=0,
$$

from (4.1),

$$
x_{2}=-\left(A_{12}\right)^{-1}\left(I+A_{11}\right) x_{1}
$$

where $\boldsymbol{x}=\left(\begin{array}{c}x_{1} \\ x_{2}\end{array}\right),\left(\boldsymbol{A}_{12}\right)_{L}^{-1}$ is the left inverse of the matrix $\left(\boldsymbol{A}_{12}\right)$, and $\boldsymbol{I}$ is the identity matrix.

The SS as (4.1) guarantees the stability of the system. One can see this in the following theorem.

Theorem 1. The sliding motion (2.6) is asymptotically stable (by Lyapunov sense) on sliding surface (4.1).

Proof. To show the stability of dynamical system (2.6) and (2.7) on SS (4.1), one can define a suitable Lyapunov function $V(x)$ from $R^{n-m}$ to $R$ as:

$$
V(x)=\frac{1}{2} x_{1}^{T} x_{1},
$$

then,

$$
\dot{V}(x)=\dot{x}_{1}^{T} x_{1}=\left(A_{11} x_{1}+A_{12} x_{2}\right)^{T} x_{1},
$$

By considering SS Equation (4.1), we have

Hence,

$$
x_{1}=-\left(A_{11} X_{1}+A_{12} X_{2}\right) \text {. }
$$




$$
\dot{V}(x)=-\left(\boldsymbol{A}_{11} x_{1}+\boldsymbol{A}_{12} x_{2}\right)^{T}\left(\boldsymbol{A}_{11} x_{1}+\boldsymbol{A}_{12} x_{2}\right)
$$

and so $V(x) \dot{V}(x)<0$.

That is, while trajectory lies on sliding surface (4.1), one have $V(x) \dot{V}(x)<0$, and this procedure, guarantees the asymptotically stability of sliding motion (2.6) with respect to SS (4.1).

\subsection{Step 2: Time Optimal Control}

Now, use of embedding technique is suggested to solve a time optimal control problem. Using this method, the sub-control $u_{a o}$ is designed such that a state trajectory starting from the initial point $B$ reaches the point $C$ on the SS in minimum time. By considering Section 3, this time optimal control problem governed by dynamical system (2.6) and (2.7) is metamorphosed to the following linear programming problem:

$$
\min \sum_{j=1}^{N} \beta_{j}
$$

subject to:

$$
\begin{array}{lll}
\sum_{j=1}^{N} \beta_{j} \Phi_{i}^{g}\left(z_{j}\right)=\Delta \Phi_{i} & i=1,2, \cdots, M_{1} & \Phi_{i} \in C^{\prime}(B) \\
\sum_{j=1}^{N} \beta_{j} \psi_{r}\left(z_{j}\right)=0 & r=1,2, \cdots, M_{2} & \psi_{r} \in D\left(J^{0}\right) \\
\sum_{j=1}^{N} \beta_{j} f_{s}\left(z_{j}\right)=a_{f s} & s=1,2, \cdots, L & f_{s} \in C_{1}(\Omega) \\
\sum_{j=1}^{N} \beta_{j} \dot{s}\left(z_{j}\right)=-s(0) & \\
\beta_{j} \geq 0, z_{j} \in \omega & j=1,2, \cdots, N . &
\end{array}
$$

The last equality guarantees the trajectory starting from $B$, hits the SS at the $C$ in minimum time

\subsection{Step 3: Equivalent Control Design}

In order to design the equivalent control, consider the SS as (4.1). The derivative of $s\left(x_{1}, x_{2}\right)=\left(\boldsymbol{I}+\boldsymbol{A}_{11}\right) x_{1}+\boldsymbol{A}_{12} x_{2}$ is as follow:

$$
\begin{aligned}
& \frac{\mathrm{d} s}{\mathrm{~d} t}=\dot{S}=\frac{\mathrm{d}}{\mathrm{d} t}\left(\left(\boldsymbol{I}+\boldsymbol{A}_{11}\right) x_{1}+\boldsymbol{A}_{12} X_{2}\right) \\
& =\dot{A}_{11} X_{1}+\left(\boldsymbol{I}+\boldsymbol{A}_{11}\right) \dot{X}_{1}+\dot{A}_{12} X_{2}+\boldsymbol{A}_{12} \dot{X}_{2}
\end{aligned}
$$

Now by considering (2.6) and (2.7), one have:

$$
\begin{aligned}
& \dot{S}=\dot{\boldsymbol{A}}_{11} X_{1}+\left(\boldsymbol{I}+\boldsymbol{A}_{11}\right)\left(\boldsymbol{A}_{11} x_{1}+\boldsymbol{A}_{12} X_{2}\right)+\dot{\boldsymbol{A}}_{12} x_{2}, \\
& +\boldsymbol{A}_{12}\left(\boldsymbol{A}_{21} X_{1}+\boldsymbol{A}_{22} X_{2}+\boldsymbol{B}_{2} u_{e q}\right)
\end{aligned}
$$

to guarantee the stability of the system on SS, we need to have, $\dot{s}=0$. So we get Equation (4.3):
This control forces the trajectories stay on the SS, and the motion is chattering free.

Remark: It is well-known that SMCs are robust against the matched disturbance and unmodelled dynamics. Note that the system (2.1) with disturbance term is

$$
\dot{x}(t)=\boldsymbol{A}(t) x(t)+\boldsymbol{B}(t) u(t)+D(t),
$$

where $D(t) \in \mathbb{R}^{n}$. Assume that the matched disturbance $D(t)$ influences the system after a certain time $t_{D}$ where $t_{s} \leq t_{D}$, and its effect on the system before this time is insignificant. Then the proposed method inherently has the robustness property because the system in the sliding mode is given by (2.6) in which the term $D(t)$ is absent. If $t_{D}<t_{s}$ one may select $t_{s}$ such that the condition $t_{s} \leq t_{D}$ is fulfilled. Then the cost functional (3.3) is minimised from $t=0$ to $t_{\mathrm{s}}$. If such information is unavailable, but the disturbance term is bound to a known function $h(t)$, i.e. $\|D(t)\| \leq h(t)$, then the term $h^{T}(t) R h(t)$ where $R$ is a positive definite symmetric matrix is included in the integrand of the cost functional (3.3). However, in the presence of disturbances, an appropriate method is to use a best estimate of $D(t)$ which is available or can be designed. Assume that $D(t)$ is a piecewise continuous and bounded signal and only a constant bound on the disturbance signal $D(t)$ may be available, i.e. there is a positive number $h_{0}$ such that $\|D(t)\| \leq h_{0}$. Let $\epsilon$ be a small positive number, two appropriate estimates of $D(t)$ are

$$
\hat{D}_{1}(t)=\left\{\begin{array}{cc}
\frac{1}{\epsilon} \int_{t-2 \epsilon}^{t-\epsilon} D(t) \mathrm{d} t & \text { if } t \geq 2 \epsilon \\
0 & \text { otherwise }
\end{array}\right.
$$

and

$$
\hat{D}_{2}(t)=\left\{\begin{array}{cl}
\max _{t-2 \epsilon \leq t \leq t-\epsilon}[D(t)] & \text { if } t \geq 2 \epsilon \\
0 & \text { otherwise }
\end{array}\right.
$$

Both estimates $\hat{D}_{1}$ and $\hat{D}_{2}$ are computable at the current time and a designer may select one of them depending on the nature of a disturbances. Therefore, in both options, the term $\hat{D}_{i}^{T}(t) R \hat{D}_{i}(t) \quad(i=1$ or $i=2)$ where $R \in \mathbb{R}^{n \times n}$ is an arbitrary positive definite symmetric matrix, is included to the integrand of the cost functional (3.3) i.e.

$$
J(x, u)=\int_{a}^{b}\left(f_{0}(t, x, u)+\hat{D}_{i}^{T}(t) R \hat{D}_{i}(t)\right) \mathrm{d} t
$$

Note that in both scenarios (4.5) and (4.6), $\left\|\hat{D}_{i}(t)\right\| \leq h_{0}$, and the cost functional (4.7) is minimised subject to

$$
\begin{gathered}
\dot{x}(t)=\boldsymbol{A}(t) x(t)+\boldsymbol{B}(t) u(t)+\hat{D}_{i}(t) \\
x(0)=x_{a}, x\left(t_{s}\right)=x_{b} .
\end{gathered}
$$

$$
u_{e q}=-\left(\boldsymbol{A}_{12} \boldsymbol{B}_{2}\right)_{L}^{-1}\left(\dot{A}_{11} x_{1}+\left(I+\boldsymbol{A}_{11}\right)\left(\boldsymbol{A}_{11} x_{1}+\boldsymbol{A}_{12} x_{2}\right)+\dot{A}_{12} x_{2}+\boldsymbol{A}_{12}\left(\boldsymbol{A}_{21} x_{1}+\boldsymbol{A}_{22} x_{2}\right)\right) .
$$




\section{Numerical Example}

Example 1. Consider the following dynamical system:

$$
\begin{gathered}
\dot{x}_{1}=x_{1}-x_{2}+2 x_{3} \\
\dot{x}_{2}=2 x_{1}-x_{2} \\
\dot{x}_{3}=3 x_{1}+x_{3}+u
\end{gathered}
$$

point.

$x(0)=(-2.25,5.25,4.2)$, and the origin is equilibrium

It is desired to design an almost time optimal control $u_{a o}$ such that the trajectory starting from the initial point $B=(-2.25,5.25,4.2)$ reaches the point $C=(-2,5,4.5)$ on the $\mathrm{SS}$ in minimum time, then derive the system from $C$ to the origin (equilibrium point) along the $\mathrm{SS}$ in an infinite time. Assume $\mathcal{A}=[-2.5,-1.5] \times[4.5,5.5] \times[4,5]$ and $\mathcal{U}=[-1.1,1.1]$. By solving the LP problem (4.2), the optimal time is found as $t_{\mathrm{s}}=0.03$. In next two steps, the SS and equivalent control $u_{e q}$ are designed such that the system is stable and the trajectories remain on the SS for all $t \geq t_{s}=0.03$.

To design SS, From (4.1) we have:

$$
x_{3}=-\left(\boldsymbol{A}_{12}\right)_{L}^{-1}\left(\boldsymbol{I}+\boldsymbol{A}_{11}\right)\left(\begin{array}{c}
x_{1} \\
x_{2}
\end{array}\right)
$$

or, $s(x)=x_{1}-0.5 x_{2}+x_{3}=0$. Now by (4.3), the equivalent control achieves as follow:

$$
u_{e q}=-3 x_{1}+0.5 x_{2}-3 x_{3} .
$$

This control causes $\dot{s}(x)=0$, so guarantee the trajectories stay on sliding surface. Now by the procedure discussed in this article, the whole trajectory functions can be found. The entire trajectories using the control

$$
u=\left\{\begin{array}{llc}
u_{a o} & \text { if } & 0 \leq t \leq t_{s} \\
u_{e q} & \text { if } & t>t_{s}
\end{array}\right.
$$

are designed through the steps 1 and 3. Figure 1 shows the action of the optimal controls (5.2), and the behaviour of the states using these controls.

Example 2. Consider the following dynamical system:

$$
\begin{aligned}
\dot{x}_{1}= & (0.15+0.02 \sin (\pi t)+0.12 \sin (10 \pi t)) x_{1} \\
& +(0.7+0.01 \cos (\pi t)) x_{2} \\
& +(0.206+0.014 \sin (2 \pi t)) x_{3} \\
\dot{x}_{2}= & (1.45+0.1 \sin (\pi t)) x_{1} \\
& +(2.1+0.05 \cos (\pi t)+0.2 \cos (5 \pi t)) x_{2} \\
& +(1.03+0.07 \sin (2 \pi t)) x_{3} \\
& \dot{x}_{3}=(-0.4+0.5 \sin (\pi t)) x_{1}+1.7 x_{2} \\
& +0.6 x_{3}+(1+0.014 \sin (\pi t)) u
\end{aligned}
$$

$x(0)=(-0.1,0.69,-1.8)$, and the origin is equilibrium point.

The above example is from [17], and is implemented by the method considered have, to compare outcome with the results appeared in [17]. It is desired to design an almost time optimal control $u_{a o}$ such that the trajectory starting from the initial point $B=(0,3,-2)$ reaches the point $C=(-0.1,0.69,-1.8)$ on the $\mathrm{SS}$ in minimum time, then derive the system from $C$ to the origin (equilibrium point) along the $\mathrm{SS}$ in an infinite time. Assume $\mathcal{A}=[-1,0] \times[-1,3] \times[-6,0]$ and $\mathcal{U}=[-1.5,1.5]$. By solving the LP problem (4.2), the optimal time is found as $t_{s}=0.88$. In next two steps, the SS and equivalent control $u_{e q}$ are designed such that the system is stable and the trajectories remain on the $\mathrm{SS}$ for all $t \geq t_{s}=0.88$.

To design SS, from (4.1) we have:

$$
x_{3}=-\left(A_{12}\right)_{L}^{-1}\left(I+A_{11}\right)\left(\begin{array}{c}
x_{1} \\
x_{2}
\end{array}\right) \text {, }
$$

hence,

$$
x_{3}=\frac{-1}{(1.03+0.07 \sin (2 \pi t))}\left((0.02 \sin (\pi t)+0.12 \sin (10 \pi t)+1.15) x_{1}+(0.01 \cos (\pi t)+0.7) x_{2}\right)
$$

and $u_{e q}$ is found from (4.3).

Figure 2 shows the action of the optimal controls (5.2), and the behaviour of the states using these controls. The trajectory hits SS at $t_{s}=0.88$ and reaches the neighbour of equilibrium point (origin) in the time less than 3 , and the behavior is chattering free. The result show that the method used in this article is more accurate than the method in [17]. Since trajectories reach SS in shorter time, nevertheless, the method is very easy to use.

Example 3. Consider the following linear dynamical system with disturbance:

$$
\begin{gathered}
\dot{x}_{1}=(-2-\sin t) x_{1}+\cos t x_{2} \\
+(1+0.1 \sin (2 \pi t)) x_{3}-\cos (3 \pi t) \\
\dot{x}_{2}=\cos t_{1}+(-2+\sin t) x_{2}+0.2 \cos (\pi t) x_{3}+2 \sin (\pi t) \\
\dot{x}_{3}=(1+\cos t-\sin t) x_{1}+2 x_{2}+\cos (t) x_{3} \\
+4 u+0.1 \sin (2 \pi t) \\
x(0)=(-2,-5,-9), \text { and the origin is equilibrium point. }
\end{gathered}
$$

Without loss of generality, we assume $x(\cdot)$ lies on SS. By (4.1) the SS defines as: 


$$
x_{3}=\frac{-K}{1+0.1 \sin (2 \pi t)}\left((\cos t+\sin t+1) x_{1}+(\sin t-\cos t-1) x_{2}\right),
$$

and from (4.3) $u_{e q}$ as:

$$
u_{e q}=-\left(\boldsymbol{A}_{12} \boldsymbol{B}_{2}\right)_{L}^{-1}\left(K \dot{\boldsymbol{A}}_{11} X_{1}+K\left(I+\boldsymbol{A}_{11}\right)\left(\boldsymbol{A}_{11} x_{1}+\boldsymbol{A}_{12} X_{2}+\rho_{1}\right)+\dot{\boldsymbol{A}}_{12} x_{2}+\boldsymbol{A}_{12}\left(\boldsymbol{A}_{21} x_{1}+\boldsymbol{A}_{22} X_{2}+\rho_{2}\right)\right),
$$

where $K$ is a design parameter and selected such that the reduced ordered system (the system in the sliding

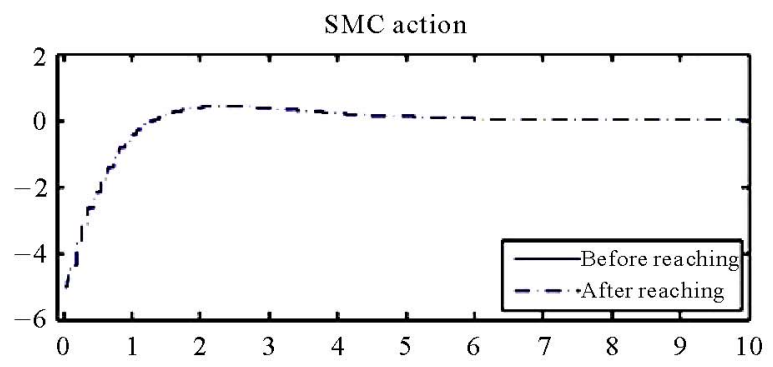

The behavior of the state $x_{2}$

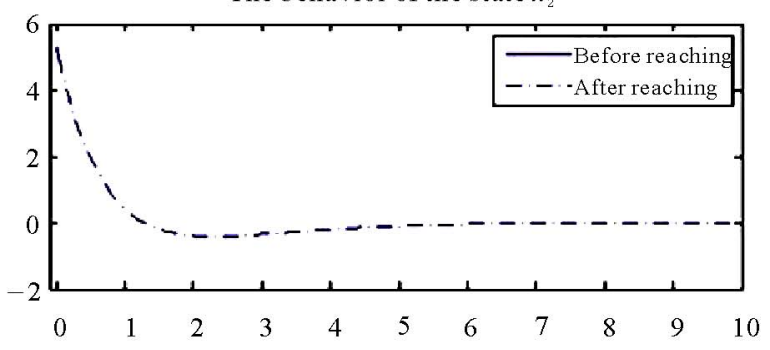

mode) to be stable. In this example, $K$ is chosen as 1.5.

Figure 3 shows the action of the controls (5.2), and

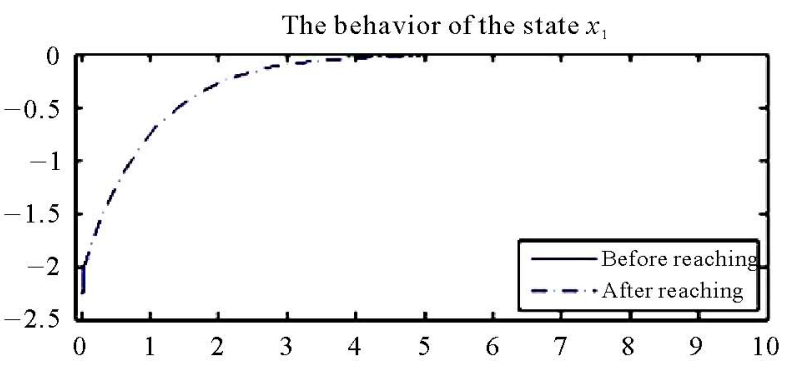

The behavior of the state $x$

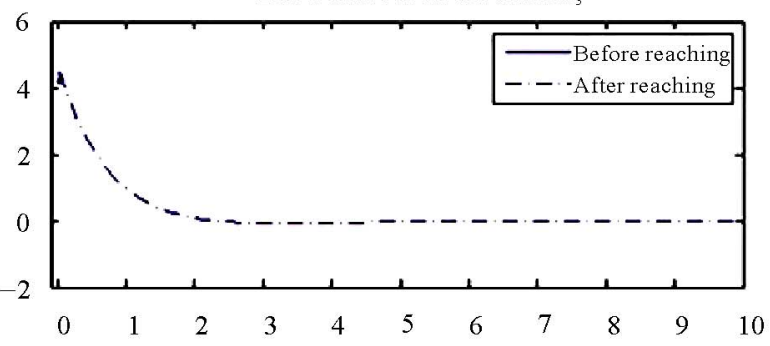

Figure 1. The action of the SMC (5.2) and the behaviour of the state trajectories using this controller for Example 1.
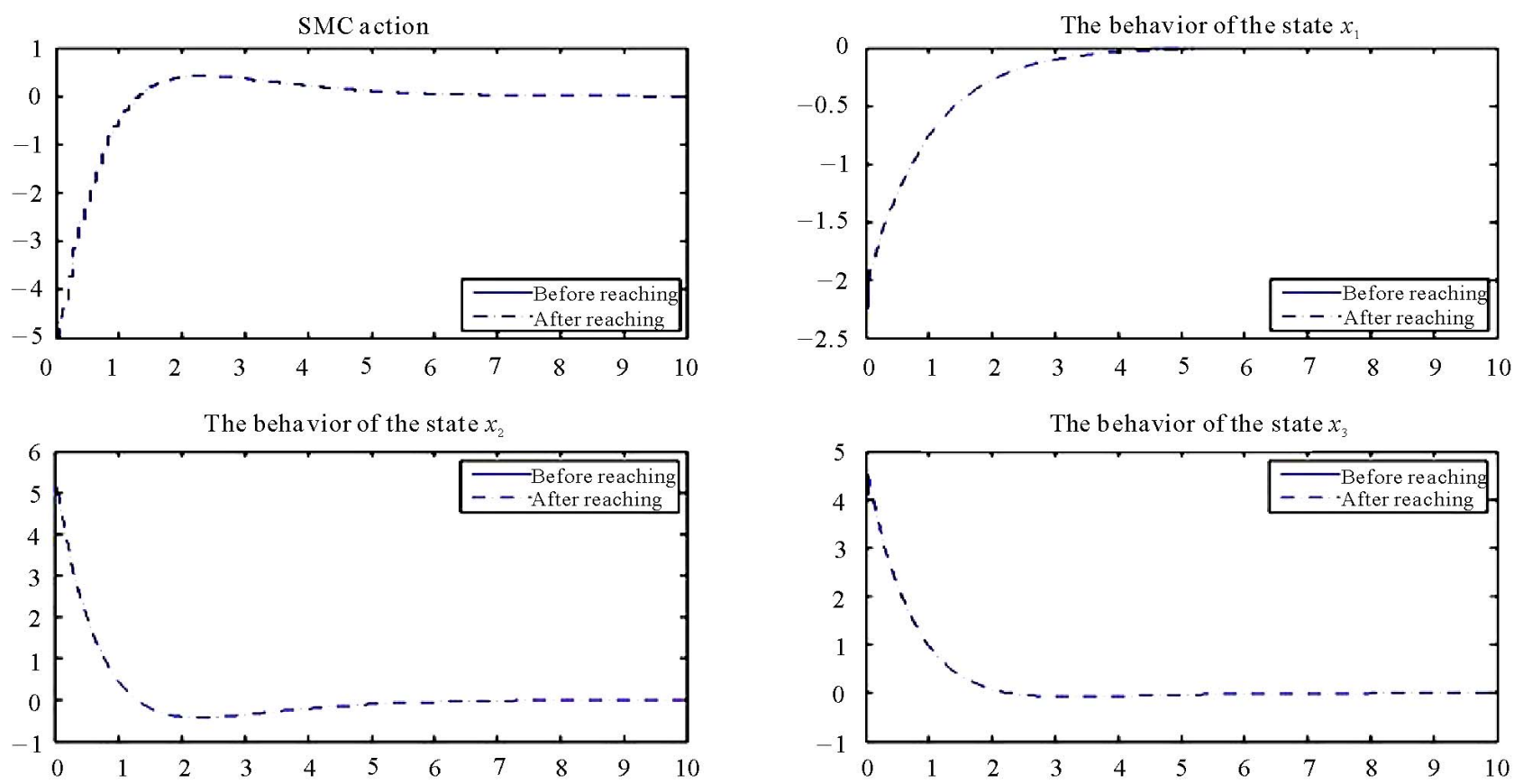

Figure 2. The action of the SMC (5.2) and the behaviour of the state trajectories using this controller for Example 2. 


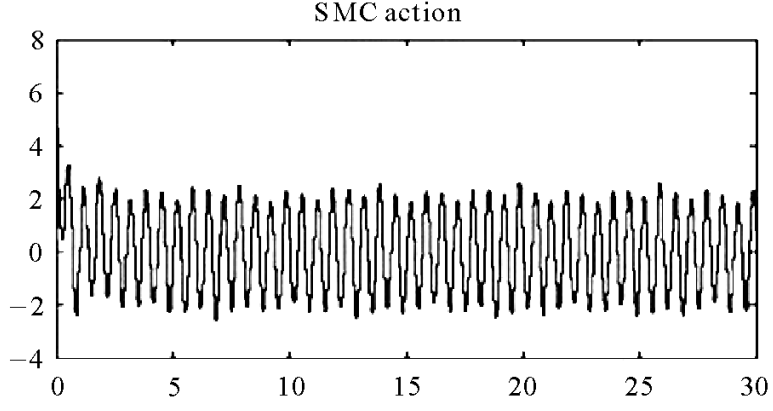

The behavior of the state $x$

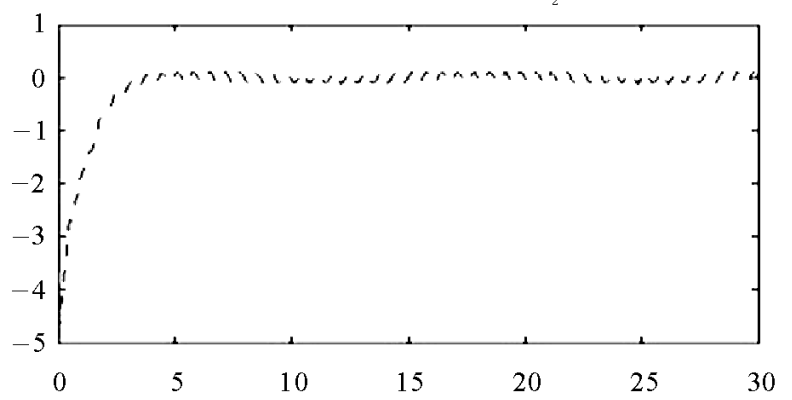

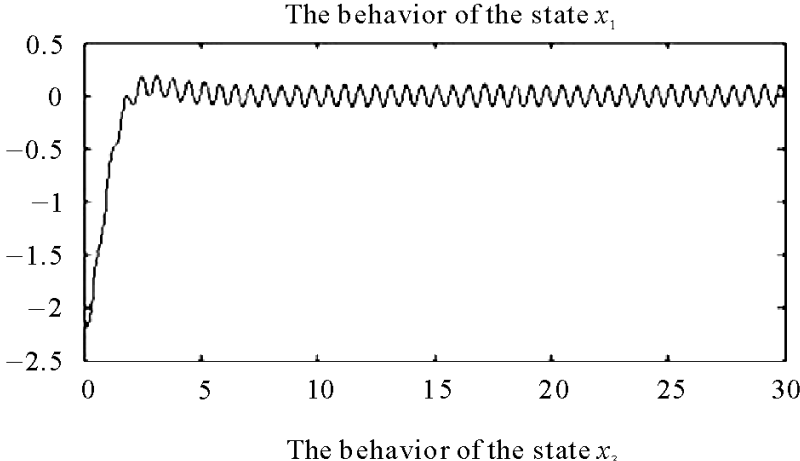

The behavior of the state $x_{3}$

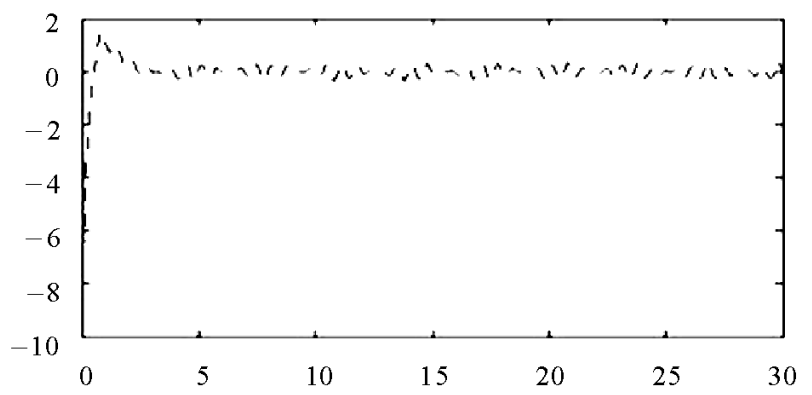

Figure 3. The action of the SMC (5.2) and the behaviour of the state trajectories using this controller for Example 3.

the behaviour of the states using these controls.

\section{Conclusions}

In this paper, a new approach based on embedding process and sliding surface to control time-varying linear dynamical systems has been proposed. The method is robust against the matched disturbances, and the sliding motion is chattering free. However, since the embedding method is independent from the linearity or non-linearity of the dynamical system, if one can design the sliding surface, the method can be applied to any linear or nonlinear system. Three numerical examples were used to support the theoretical results, and show the effectiveness and reliability of the proposed method.

\section{References}

[1] S. V. Emeyanov, et al., "Variable Structure Control Systems," Mir Publishers, Moscow, 1967.

[2] I. Flügge-Lotz, "Discontinuous Automatic Control," Princeton University Press, New Jersey, 1953.

[3] A. F. Filippov, "Application of Theory of Differential Equations with Discontinuous Right-Hand Side to Nonlinear Control Problems," Stability of Stationary Sets in Control Systems with Discontinuous Nonlinearities, World Scientific Publishing, Moscow, 1960.

[4] V. I. Utkin, "Sliding Modes in Control and Optimization," Springer-Verlag, Berlin, 1992.

[5] S. V. Emeyanov and V. A. Taran, "On One Class of Variable Structure Control Systems," Computational Mathe- matics and Modeling, Vol. 21, No. 3, 2006, pp. 5-26.

[6] B. Draženović, "The Invariance Condition in Variable Structure Systems," Automatica, Vol. 5, No. 3, 1969, pp. 287-295.

[7] U. Itkis, "Control System of Variable Structure," Wiley, New York, 1976.

[8] V. I. Utkin, "Variable Structure Systems with Sliding Modes," IEEE Transactions on Automatic Control, Vol. 22, No. 2, 1977, pp. 212-222. doi:10.1109/TAC.1977.1101446

[9] T. Chatchanayuenyong and M. Parnichkun, "Neural Network Based-Time Optimal Sliding Mode Control for an Autonomous Underwater Robot," Mechatronics, Vol. 16, No. 8, 2006, pp. 471-478.

doi:10.1016/j.mechatronics.2006.02.003

[10] A. J. Koshkouei, "Sliding-Mode Control with Passivity for a Continuously Stirred Tank Reactor," Proceedings of the Institution of Mechanical Engineers, Vol. 221, No. 5, 2007, pp. 749-755.

[11] J. Mozaryn and J. E. Kurek, "Design of Decoupled Sliding Mode Control for the PUMA 560 Robot Manipulator," Proceedings the 3rd International Workshop on Robot Motion and Control, Warsaw, 9-11 November 2002, pp. 45-50.

[12] N. Yagiz Y. Z. Arslan and Y. Hacioglu, "Sliding Mode Control of a Finger for a Prosthetic Hand," Vibration and Control, Vol. 13, No. 6, 2007, pp. 733-749.

[13] A. Nowacka-Leverton and A. Bartoszewicz, "IAE Optimal Sliding Mode Control of Cable Suspended Loads," CEAI, Vol. 10, No. 3, 2008, pp. 3-10.

[14] H. Bouadi and M. Tadjine, "Nonlinear Observer Design and Sliding Mode Control of Four Rotors Helicopter," 
Vorld Academy of Science, Engineering and Technology, Vol. 25, 2007, pp. 225-229.

[15] A. S. I. Zinober, "Variable Structure and Lyapunov Control," Springer Verlag, London, 1994. doi:10.1007/BFb0033675

[16] J.-C. Le, and Y.-H. Kuo, "Decoupled Fuzzy Sliding Mode Control," IEEE Transactions on Fuzzy Systems, 1998, Vol. 6, No. 3, pp.426-435. doi:10.1109/91.705510

[17] S. H. Jang and S. W. Kim, "A New Sliding Surface Design Method of Linear Systems with Mismatched Uncertainties," IEICE Transactions on Fundamentals of Electronics, Vol. E88-A, No. 1,2005, pp. 387-391.

[18] B. Bandyopadhyay and S. Janardhanan, "Discrete-Time Sliding Mode Control," Springer-Verlag, Berlin, 2005.

[19] M. Thoma, F. Allgöwer and M. Morari, "Time-Varying Sliding Modes for Second and Third Order Systems," Springer-Verlag, Berlin, 2009.

[20] A. J. Koshkouei, "Passivity-Based Sliding Mode Control for Nonlinear Systems," International Journal of Adaptive Control and Signal Processing, Vol. 22, No. 9, 2008, pp. 859-874. doi:10.1002/acs.1028

[21] A. J. Koshkouei, K. Burnham and A. S. I. Zinober, "Flatness, Backstepping and Sliding Mode Controllers for Nonlinear Systems," In: G. Bartolini, L. Fridman, A. Pisano and E. Usai, Eds., Modern Sliding Mode Control Theory, Springer-Verlag, Berlin, 2008, pp. 269-290. doi:10.1007/978-3-540-79016-7 13

[22] B. Friedland, "Advanced Control System Design," Prentice-Hall, Englewood Cliffs, 1996.

[23] A. J. Koshkouei and A. S. I. Zinober, "Sliding Mode Controller-Observer Design for SISO Linear Systems," International Journal of Systems Science, Vol. 29, No. 12, 1998, pp. 1363-1373. doi:10.1080/00207729808929622

[24] S. H. Żak and S. Hui, "On Variable Structure Output Feedback Controllers for Uncertain Dynamic Systems," IEEE Transactions on Automatic Control, Vol. 38, No. 10, 1993, pp. 1509-1512. doi:10.1109/9.241564

[25] R. El-Khazali and R. DeCarlo, "Output Feedback Variable Structure Control Design," Automatica, Vol. 31, No. 6, 1995, pp. 805-816. doi:10.1016/0005-1098(94)00151-8

[26] C. Edwards and S. K. Spurgeon, "Sliding Mode Control: Theory and Applications," Taylor \& Francies, London, 1998.

[27] J. E. Rubio, "Control and Optimization; The Linear Treatment of Nonlinear Problems," Manchester University Press, Manchester, 1986.

[28] W. Rudin, "Real and Complex Analysis," 3rd Edition, McGraw-Hill, New York, 1987.

[29] S. Effati, A. V. Kamyad and R. A. Kamyabi-Gol, "On Infinite-Horizon Optimal Control Problems," Journal for Analysis and Its Applications, Vol. 19, No. 1, 2000, pp. 269-278. 